\title{
DINÁMICA DE SISTEMAS, UNA HERRAMIENTA PARA LA EDUCACIÓN AMBIENTAL EN INGENIERÍA
}

\author{
Danny W. Ibarra Vega ${ }^{1}$ \\ Johan Manuel Redondo ${ }^{2}$
}

Recibido el 10 de junio de 2014, aprobado el 27 de noviembre de 2014 y actualizado el 29 de abril de 2015

DOI: 10.17151/luaz.2015.41.9

\section{RESUMEN}

Este trabajo presenta parte de la experiencia acumulada en la utilización de la Dinámica de Sistemas, como herramienta para la educación ambiental de estudiantes de ingeniería de la Universidad Sergio Arboleda. A partir de la Dinámica de Sistemas, se recrean diversos escenarios que permiten al estudiante tener una percepción sistémica del mundo real y visualizar su dinámica. Dado que el medio ambiente es un sistema complejo, la utilización de la Dinámica de Sistemas favorece la identificación de relaciones no lineales entre los atributos ambientales, la modelización y la extensión a otras técnicas de comprensión como lo es la teoría matemática de los sistemas dinámicos, favoreciendo así la inclusión de lo ambiental de forma transversal en el currículo, pero además, permitiendo la apropiación de elementos indispensables en el ejercicio profesional de un ingeniero. De este modo, presentamos tres ejemplos de trabajos con Dinámica de Sistemas que se pueden implementar con estudiantes de ingeniería para introducir conceptos ambientales, que fueron desarrollados en la línea de investigación de Ciencias de la Complejidad y Medio Ambiente de la Universidad Sergio Arboleda, con algunos de sus estudiantes de ingeniería. Se concluye que la utilización de la Dinámica de Sistemas en la enseñanza de conceptos ambientales, actúa como pauta que conecta diferentes campos del conocimiento requeridos para la comprensión de la situación ambiental actual del planeta, convirtiéndose así en una herramienta para la transdisciplinariedad en ingenierías.

\section{PALABRAS CLAVE}

Dinámica de Sistemas, educación ambiental, ingeniería.

\section{SYSTEM DYNAMICS, A TOOL FOR ENVIRONMENTAL EDUCATION IN ENGINEERING}

\begin{abstract}
This paper presents part of the experience gained in using system dynamics as a tool for environmental education of engineering students from Universidad Sergio Arboleda. From, diverse scenarios that allow students to have a systemic view of the real world and visualize its dynamics, System Dynamics are recreated. Since the environment is a complex system, using
\end{abstract}


System Dynamics favors the identification of nonlinear relationships between the environmental attributes, modeling and extension to other techniques such as understanding the mathematical theory of systems dynamic, thus contributing to the inclusion of environmental issues in a cross-curricular manner, but also allowing the appropriation of the necessary elements in the professional exercise as an engineer. This way, three examples of work with system dynamics are presented which can be implemented with engineering students to introduce environmental concepts that were developed by the research line of Sciences of Complexity and Environment of Universidad Sergio Arboleda, with some of their engineering students. It is concluded that the use of System Dynamics in the teaching of environmental concepts acts as a guideline that connects different fields of knowledge required for understanding the planet's current environmental situation, thus becoming a transdisciplinary tool in engineering.

\section{KEY WORDS}

System Dynamics, environmental education, engineering.

\section{INTRODUCCIÓN}

La Dinámica de Sistemas es una metodología para el análisis y resolución de problemas, desarrollada por Jay Forrester y presentada en sus obras (Forrester, 1999a; 1999b). En Dinámica de Sistemas, se concibe cualquier aspecto del mundo como la interacción causal entre atributos que lo describen. De esta forma, se construyen representaciones sistémicas con flechas y puntos, denominadas diagramas causales, que capturan todas las hipótesis propuestas por el modelador, desde las que se puede aprender del sistema para intervenir sobre él en el ejercicio de decisión. La Dinámica de Sistemas en el área ambiental tiene su primer y principal antecedente cuando se aplicó a finales de los sesenta en un estudio conocido como "Los límites al crecimiento" presentado ante el Club de Roma. El Club de Roma, una asociación privada compuesta principalmente por empresarios, científicos y políticos, designó a un grupo de investigadores para realizar esta investigación. Sus resultados fueron la base del primer informe en el que se realizaron proyecciones de la población, los recursos disponibles y la contaminación del planeta. Este modelo, tuvo gran incidencia en la opinión pública y fue objeto de múltiples debates, lo cual contribuyó a la difusión de la Dinámica de Sistemas (Aracil y Gordillo, 1997). En el informe del Club de Roma se llegó a la conclusión de que:

[...] si la industrialización, la contaminación ambiental, la producción de alimentos y el agotamiento de los recursos mantienen las tendencias actuales de crecimiento de la población mundial, este planeta alcanzará los límites de su crecimiento en el curso de los 
próximos cien años. El resultado más probable sería un súbito e incontrolable descenso, tanto de la población como de la capacidad industrial. (Meadows et al., 1972 pg 182)

La Figura 1 presenta los resultados de sus simulaciones:

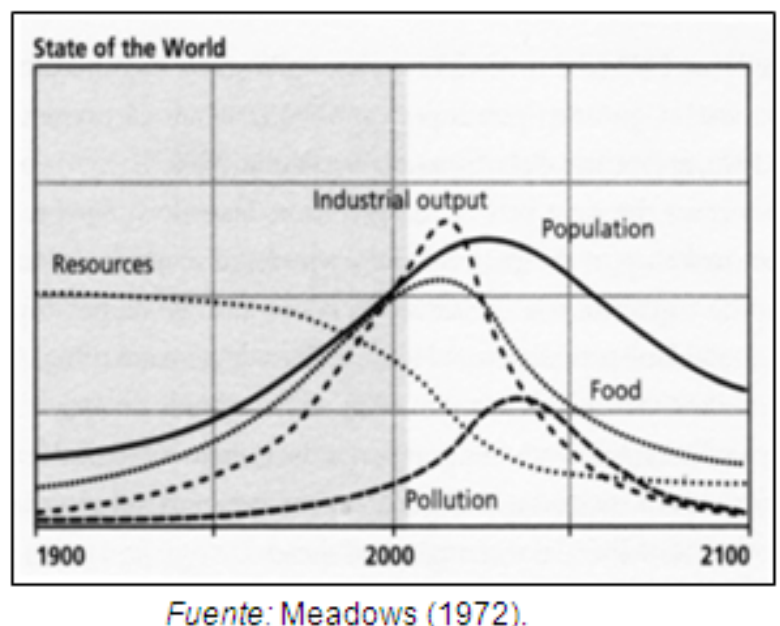

Figura 1. Límites al Crecimiento.

En la Figura 1 vemos que una disminución en la tasa de extracción de recursos no renovables por el agotamiento de los mismos, dará lugar a una disminución de la producción mundial de alimentos y de la producción industrial, que a su vez conduciría a la disminución de los niveles de vida para todos.

\section{La educación y Dinámica de Sistemas}

La educación y la enseñanza usualmente utilizan problemas lineales y estáticos del mundo real. Difícilmente logra mostrar los problemas del mundo que son dinámicos. Forrester establece en una publicación sobre educación que: "La mente humana toma imágenes, mapas y relaciones estáticas de una manera maravillosamente efectiva" (Forrester, 1992 pg 6). Pero cuando los sistemas y sus partes interactúan, esa mente humana no es buena para simular y para percibir los cambios a través del tiempo del sistema.

De esta forma, se requiere de un modelo de aprendizaje para generar capacidad de adaptación y cambio ante los retos de ambiente externos, complejos y dinámicos (Senge, 1993, 2006). Así la dinámica de sistemas se ha venido utilizando como herramienta pedagógica en diferentes lugares del mundo, ya que ofrece un marco para dar cohesión, significado y motivación a la educación en todos los niveles, desde la educación primaria en adelante (Forrester, 1992).

De igual manera, se han diseñado programas informáticos que permiten la implementación de la Dinámica de Sistemas que han 
sido la propuesta de inclusión del modelado y simulación en los procesos pedagógicos de la educación básica y media (Andrade, Maestre y Gómez, 2008). La propuesta que se plantea es mostrar que la Dinámica de Sistemas es un instrumento que facilita la integración transversal de conceptos ambientales para la formación de ingenieros, por medio de la modelización de sus problemas, mas no como una nueva asignatura en el currículo.

\section{IMPLEMENTACIÓN DE MODELOS AMBIENTALES}

A continuación presentamos algunos ejemplos de modelación utilizando Dinámica de Sistemas en diferentes niveles de profundidad, que fueron construidos en discusiones con estudiantes de ingeniería de la Universidad Sergio Arboleda y la participación de estudiantes de la especialización en Gerencia Ambiental y Prevención de Desastres de la misma universidad.

La metodología que se sigue en la construcción de los modelos ambientales que se presentan a continuación es la siguiente: las discusiones inician con una pregunta de investigación y la posterior identificación de los atributos del sistema que permitirían resolver la pregunta. Se consideran entonces las relaciones causales entre los atributos, teniendo cuidado de que los argumentos que las justifican estén bien conceptualizados. El resultado es el diagrama causal. Acto seguido, se consideran los atributos del diagrama causal como variables de estado, razones de cambio, variables auxiliares y parámetros del sistema [véase una discusión más profunda en Aracil y Gordillo (1997) o Sterman (2000)] y se construye el diagrama de niveles y flujos. Con el diagrama de niveles y flujos se discute la expresión matemática que representaría los comportamientos del sistema, teniendo cuidado en el uso de las unidades. Finalmente, se utilizan programas especializados de simulación para visualizar el sistema y sacar algunas conclusiones.

El resultado del ejercicio no apunta a la predicción, sino al aprendizaje del sistema, quedando al descubierto comportamientos del sistema que mejoran la fundamentación ambiental de los estudiantes, en problemas específicos como efecto invernadero, residuos y capacidad de soporte del incremento poblacional.

\section{Emisiones de gases de efecto invernadero (GEI)}

En este ejemplo construido en clase se trata de representar la pregunta: ¿Cuál podría ser un efecto de los programas de reducción de emisiones de gases de efecto invernadero sobre el cambio climático? De esta forma el diagrama causal propuesto para el modelo se presenta la Figura $2 a$, y se lee de la siguiente manera: a mayores emisiones de Gases Efecto Invernadero a la atmosfera, habrá un aumento en el calentamiento global. El cual de acuerdo a convenios internacionales como el Protocolo de Kioto, incentivará la puesta en marcha de proyectos de reducción de emisiones, de este modo habrá una disminución de las 
emisiones de GEI, pero no será una eliminación de las fuentes de emisiones. Solo representará una disminución de GEI.

a

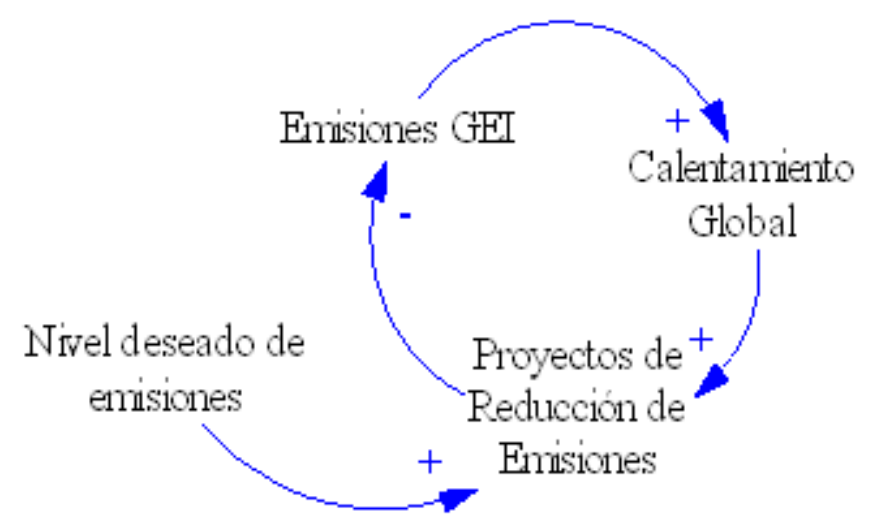

b

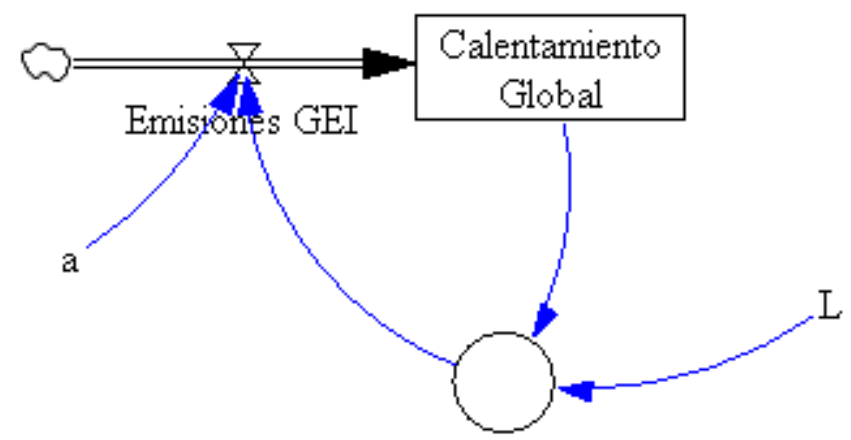

Proyectos de reducción de emisiones

Fuente: Ios autores.

Figura 2. a) Arriba, el diagrama causal.

b) Abajo, el diagrama de Niveles y Flujos.

En este sistema se acumula la temperatura, razón por la que hemos tomado el calentamiento global $\boldsymbol{x}$ en términos de temperatura como variable de estado. El calentamiento es producido por las emisiones de GEI que se considera entonces la razón de cambio del sistema. Los proyectos de reducción de emisiones $\boldsymbol{P}$ se tomaron como variable de decisión o variable auxiliar del sistema. Se consideraron los parámetros a y $L$ que corresponden a la tasa de cambio de las emisiones de GEI y a un nivel de referencia de las emisiones, respectivamente. Las relaciones entre las variables se expresan como funciones matemáticas, como se presenta a continuación: 


$$
\frac{d x}{d t}=\text { Emisiones GEI }
$$$$
\text { Emisiones de } G E I=a / P
$$

$$
P=L+x
$$

Remplazando la ecuación de flujo (1) y la auxiliar (2) en la ecuación de Nivel (3), queda entonces conformada una sola ecuación denominada ecuación de Estado (4), que representará el comportamiento del sistema en el tiempo $\boldsymbol{t}$ :

$$
\frac{d x}{d t}=\frac{a}{L+x}
$$

A continuación se presentan los resultados de la aplicación del modelo (ver Figura 3) que nos muestran a través de las simulaciones cómo es el comportamiento de las variables.

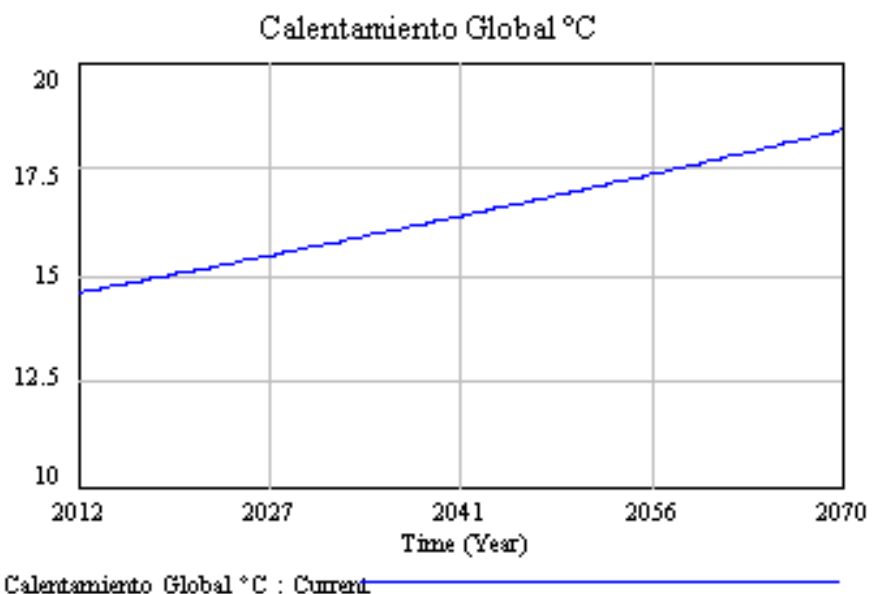

Fuente: los autores.

\section{Figura 3. Simulación del Modelo de Cambio Climático.}

Como discusión vemos que los programas de reducción de emisiones de GEI pueden influenciar la velocidad a la que el planeta se calienta, pero no eliminar los GEI de la atmósfera o detener el calentamiento que estamos sufriendo. Se ve el incremento de la temperatura desde $14,6^{\circ} \mathrm{C}$ en 2012 hasta un valor cercano a los $18^{\circ} \mathrm{C}$ en 2072 . Esto permite sensibilizar al estudiante sobre la necesidad de proponer mecanismos más efectivos que se sumen al esfuerzo de los programas de reducción de emisiones de GEI y considerarlo en un nuevo modelo para evaluar sus implicaciones. Este ejemplo le permite al estudiante evidenciar que el problema ambiental global requiere de múltiples acciones y no solamente de la 
transformación tecnológica propuesta por los programas de reducción de emisiones de GEI.

\section{Residuos sólidos y población}

A continuación se presenta un modelo de residuos sólidos desarrollado por Redondo y Solano (2010) que trataba de responder a la pregunta: ¿Cuál es la incidencia de la población sobre la generación de residuos y la calidad ambiental? En las Figuras 4 y 5 se muestra el diagrama causal y el diagrama de Niveles y Flujos, en donde se presentan las principales relaciones existentes entre los atributos de la tríada: población, generación de residuos y calidad ambiental.

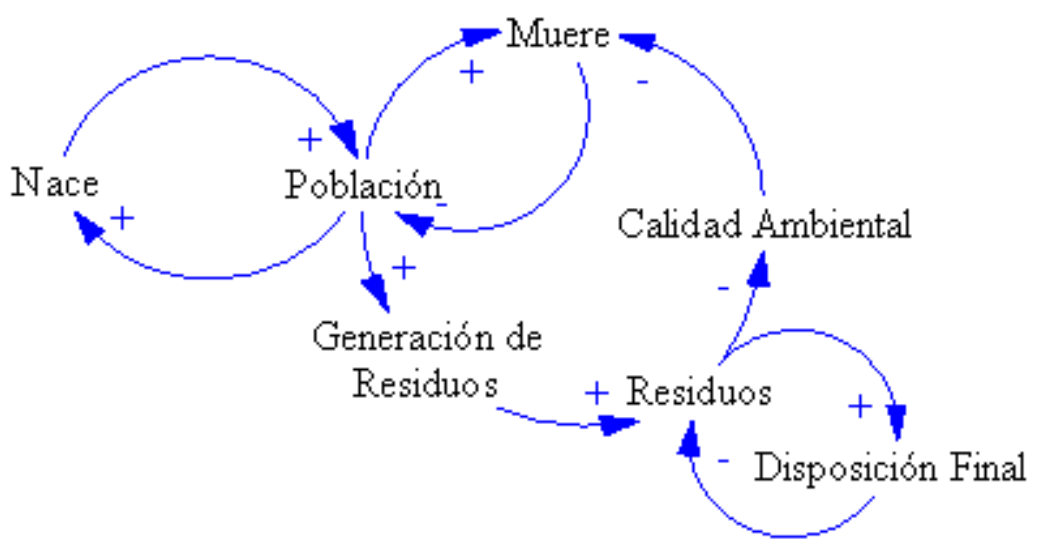

Fuente: Redondo y Solano (2010)

Figura 4. Diagrama Causal de Residuos. 


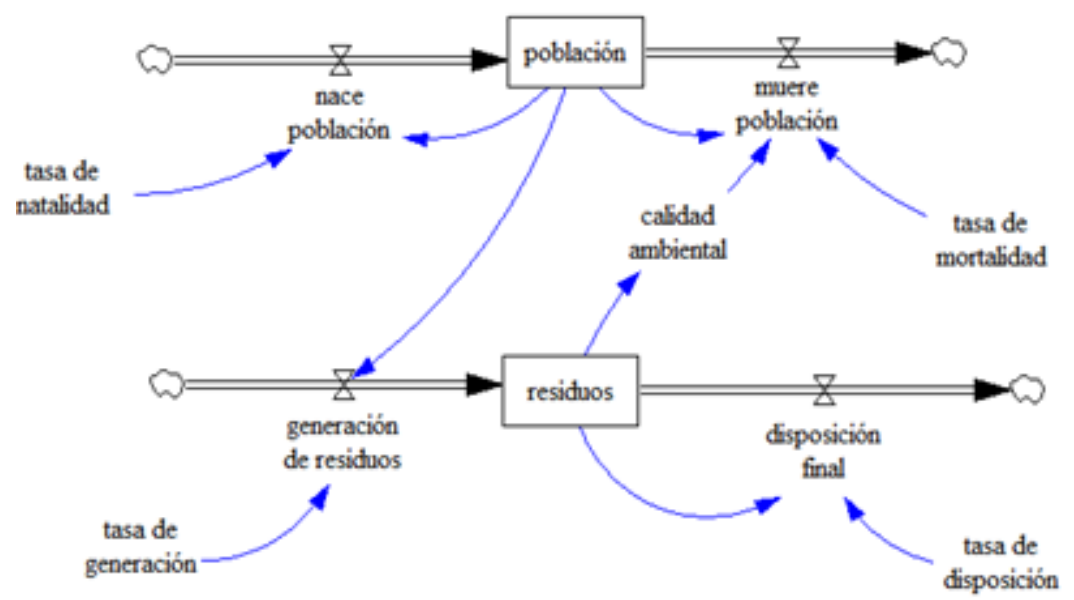

Fuente: los autores.

Figura 5. Diagrama de Niveles y Flujos de Residuos.

En este sistema se acumula la población y la de residuos generados, razón por la cual las tomamos como variables de estado como número de personas y cantidad de residuos. Estos se incrementan debido a la generación de residuos por parte de la población, esta generación de residuos se considera entonces la razón de los residuos generados. La calidad ambiental es una variable auxiliar que está en función de los residuos sólidos. Se consideran como parámetros: tasa de natalidad, mortalidad, generación de residuos y tasa disposición. En la Figura 6 se presenta el espacio de fase del sistema, en el cual resaltó la trayectoria con condiciones iniciales $X(0)=500, Y(0)=7500$. 
a

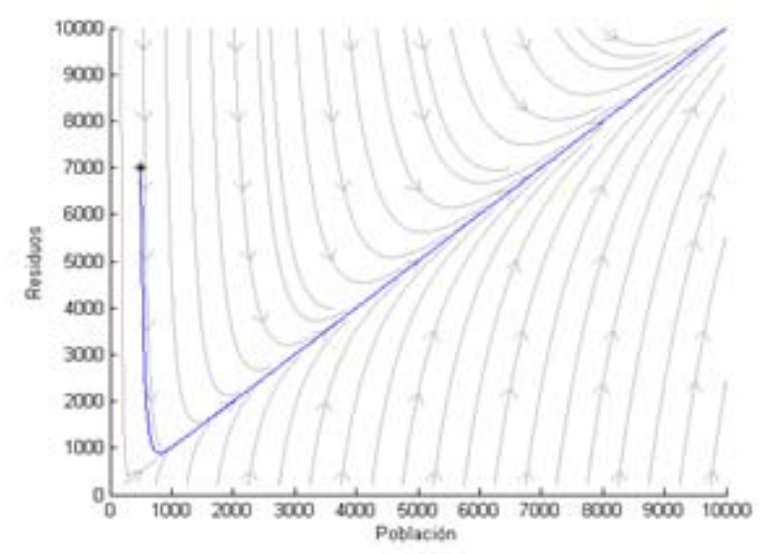

b
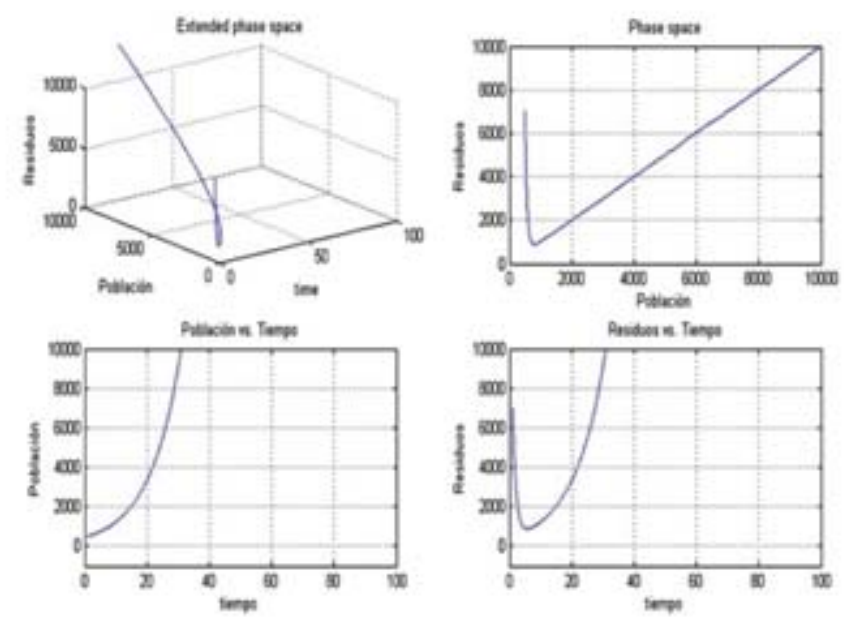

Fuente: los autores.

\section{Figura 6. a) Espacio de fases.}

b) Evolución temporal de las variables.

Tomada la hipótesis de una relación inversa entre calidad ambiental y residuos acumulados, evidenciamos una dinámica muy interesante entre la población y sus residuos. Tal dinámica muestra dos intenciones:

En la primera (condiciones iniciales por encima de la variedad inestable), los residuos disminuyen, aumentando la calidad ambiental, la cual a su vez favorece las condiciones para el incremento de la población. Pero al incrementarse la población, aumentan linealmente los residuos que estos generan, desmejorando su calidad ambiental.

En la segunda (condiciones iniciales por debajo de la variedad inestable), tanto la población como sus residuos crecen hasta alcanzar la recta de equilibrio en donde aumentan conjuntamente de forma lineal.

Estos argumentos le permiten al estudiante justificar la necesidad de implementar tecnologías dentro de la industria, para la 
disposición adecuada de los residuos y la transformación cultural de nuestra sociedad de consumo, con el fin de sensibilizar a los futuros profesionales que, el día de mañana, harán parte de la industria.

\section{Población con capacidad de soporte}

La pregunta de investigación en este último ejemplo es: ¿Cómo se relacionan la población y la capacidad de soporte en el planeta? Esta es una pregunta sencilla, pero sus implicaciones técnicas y las conclusiones son muy atractivas para los estudiantes. El diagrama causal propuesto para este problema se presenta en la Figura 7a y se lee de la siguiente manera:

Los nacimientos incrementan la población que a su vez influye en el aumento de nacimientos y en la disminución de la disponibilidad de recursos. Suficientes recursos se reflejan en el aumento de los nacimientos en la población. El aumento de la población aumentará la cantidad de muertes para un cierto tiempo, lo que conduce a su vez a la disminución de la población. De este modo, se conforman dos bucles de realimentación negativos y uno positivo.

a

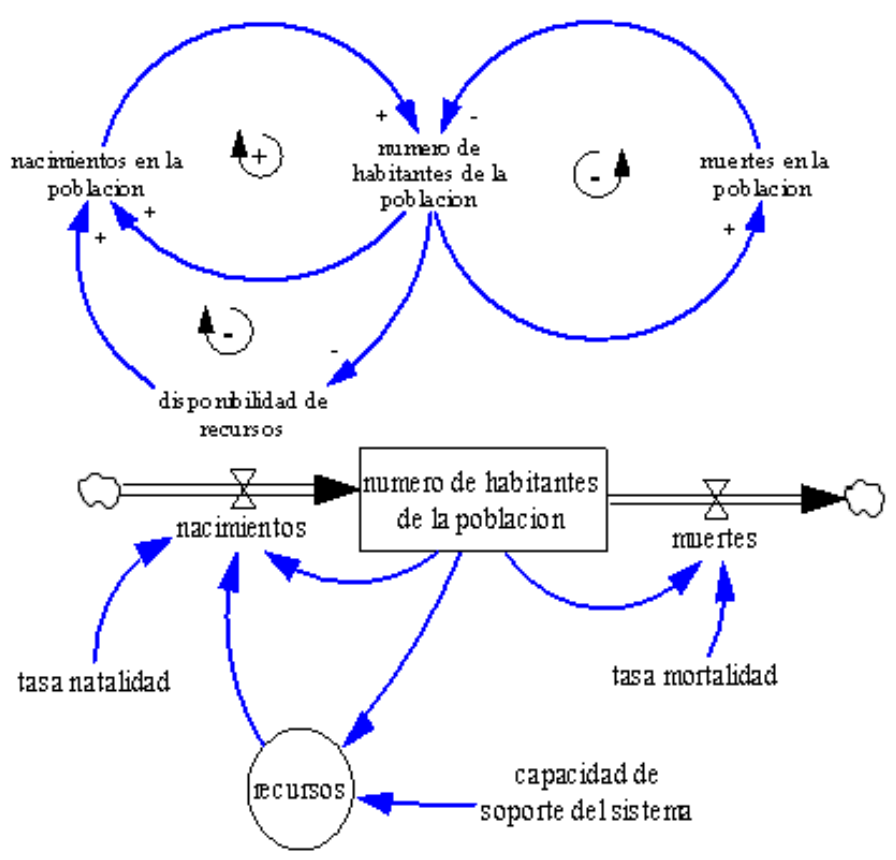

Fuente: Ios autores.

Figura 7. a) Diagrama causal Capacidad de Soporte.

b) Diagrama de Niveles y Flujos Capacidad de Soporte.

En este sistema se acumula el número de individuos de la población $\boldsymbol{x}$, convirtiéndose así en la variable de estado. El número de habitantes depende de los nacimientos y muertes en el sistema, por lo que se han considerado como razones de cambio. Los recursos naturales se tomaron como variable de 
decisión o variable auxiliar del sistema a la que se le ha asociado el parámetro capacidad de soporte $\boldsymbol{L}>\mathbf{0}$ del sistema. También se consideraron los parámetros tasa de natalidad $\mathbf{a}>\mathbf{0}$ y tasa de mortalidad $\boldsymbol{b}>\mathbf{0}$.

Las relaciones entre las variables se expresan como se presenta a continuación:

$$
\begin{aligned}
& \frac{d x}{d t}=\text { nacimientos }- \text { muertes; } \\
& \text { nacimientos }=a * \text { Recursos } * x ; \\
& \text { Muertes }=b * x ; \\
& \text { Recursos }=1-x / L
\end{aligned}
$$

Remplazando la ecuación de flujo (6) y la auxiliar (7) en la ecuación de Nivel (5), obtenemos el modelo matemático que representará el comportamiento del sistema en el tiempo:

$$
\frac{d x}{d t}=a x\left(1-\frac{x}{L}\right)-b x
$$

Siguiendo la teoría de los sistemas dinámicos, vemos que esta ecuación diferencial tiene dos puntos de equilibrio: la población nula $(x=0)$, que es un punto de equilibrio inestable, y el valor positivo de la población $x=L(1-a / b)$, que es un punto de equilibrio estable del sistema. Las ecuaciones son las siguientes:

$$
\begin{aligned}
& x=0 \\
& x=L(1-b / a)
\end{aligned}
$$

Con la ecuación 10 y 11 se logran construir los diagramas de bifurcación que se muestran en la Figura 8 ( $a, b$ y c). Los diagramas de bifurcación permiten ver la variación del comportamiento dinámico del sistema con respecto a la variación de parámetros. En este sistema, dado que los parámetros y la población se consideran cantidades no negativas, no se aprecian puntos de bifurcación, sin embargo, se puede visualizar el escenario del sistema. 


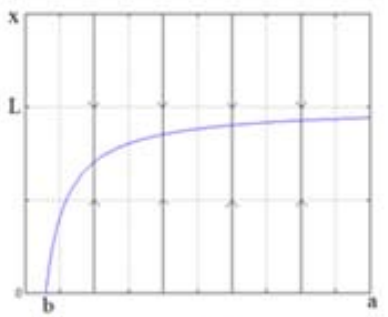

a

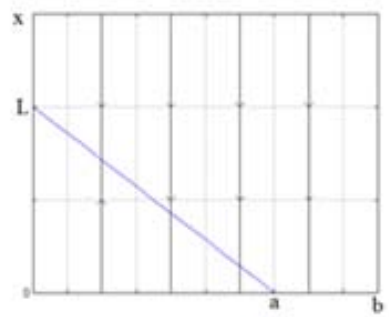

b

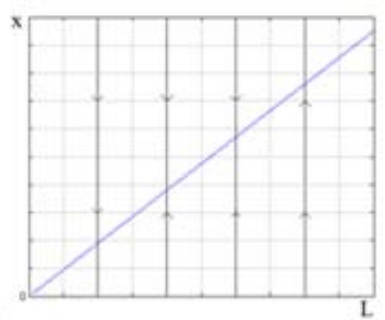

C

Fuente: los sutores.

Figura 8. Diagrama de bifurcaciones. a) Variación con respecto a la tasa de natalidad, b) con respecto a la tasa de mortalidad, y c) con respecto a la capacidad de soporte.

Cuando la tasa de natalidad es mayor que la tasa de mortalidad la población crece, pero no tanto como para superar la capacidad de soporte del sistema (Figura 8, a y b), de ahí que si se considera un crecimiento de la población, debe considerarse también la expansión de la capacidad de soporte del sistema (Figura 8, c), pero esto no es posible porque nuestro planeta tiene recursos limitados.

Este ejemplo induce la discusión sobre los patrones de consumo y la sobrepoblación en un planeta finito, conduciendo al estudiante de ingeniería al desarrollo de una conciencia de sus vanidades y aspiraciones.

\section{CONCLUSIONES}

Se concluye que la utilización de la Dinámica de Sistemas en la enseñanza de conceptos ambientales, actúa como pauta que conecta diferentes campos del conocimiento requeridos para la comprensión de la situación ambiental actual del planeta, convirtiéndose así en una herramienta para la transdisciplinariedad.

Estos modelos desarrollados en el marco de una actividad académica, aportan y procuran el mejoramiento de la calidad de la educación ambiental en diferentes áreas, ya que estos recrean y simulan comportamientos de interés en el desarrollo curricular de las ingenierías, buscando darle una mayor rigurosidad en términos de conocimiento al componente ambiental que debe ser interdisciplinar.

\section{REFERENCIAS}

- Andrade, H., Maestre, G. y López, G. (2008). La lúdica y las redes humanas como estrategia para promover la sostenibilidad de la incorporación de la dinámica de sistemas en las escuelas colombianas. VI encuentro Colombiano de Dinámica de Sistemas. 
- Aracil, J. y Gordillo, F. (1997). Dinámica de Sistemas. Madrid: Alianza Editorial S.A.

- Forrester, J. (1992). La Dinámica de Sistemas y el Aprendizaje del Alumno en la educación escolar. Proyecto Educativo Dinámica de Sistemas. Grupo de Dinámica de Sistemas Escuela de Administración Massachusetts Institute of Technology.

- . (1999a). Industrial Dynamics. Waltham: Pegasus Communications, Inc.

- $\quad$ (1999b). Urban Dynamics. Waltham: Pegasus Communications, Inc.

- Meadows, D.H., Meadows, D.L., Randers, J. y Behrens, W. (1972). Los límites del crecimiento: informe al Club de Roma sobre el predicamento de la Humanidad. Fondo de Cultura Económica.

- Redondo, J. y Solano, J. (2010). Dinámica de sistemas aplicada a la triada: población, generación de residuos y calidad ambiental. Una perspectiva que integra: la dinámica de sistemas y los sistemas dinámicos. VIII Congreso Latinoamericano de Dinámica de Sistemas.

- Senge, P. (1993). La quinta disciplina. Buenos Aires: Granika.

- (2006). La Quinta Disciplina, estrategias y herramientas para construir la organización abierta al aprendizaje. Buenos Aires: Granica.

- Sterman, J. (2000). Business Dynamics, Systems thinking and modelling for a complex world. McGraw-Hill.

1. Ingeniero Biotecnológico. Especialista en Gerencia del Medio Ambiente y Prevención de Desastres. MSc. en Gestión Ambiental. Docente e Investigador, Universidad Sergio Arboleda. danny.ibarra@usa.edu.co

2. Ingeniero Ambiental. Especialista en Matemática Aplicada. Magíster en Docencia e Investigación Universitaria. Doctorado en Ingeniería. Docente e Investigador, Universidad Sergio Arboleda. galileonp@ima.usa.edu.co

Para citar este artículo: Ibarra Vega, D.W. y Redondo, J.M. (2015). Dinámica de Sistemas, una herramienta para la educación ambiental en ingeniería. Revista Luna Azul, 41, 152$164 . \quad$ Recuperado de http://lunazul.ucaldas.edu.co/index.php?option=content\&tas $k=$ view\&id=1060 